\title{
Magnetic Flux Leakage Nondestructive Testing Technology of High-Speed Rail Defects
}

\author{
Haixia Zhu1,2, a, Wenbo Liü,b \\ ${ }^{1}$ NANHANG JINCHENG COLLEGE, Nanjing, China \\ ${ }^{2}$ College of Automatic Engineering Nanjing University of Aeronautics and Astronautics, \\ Nanjing, China \\ azhuhaixia@nuaa.edu.cn, bwenboliu@nuaa.edu.cn
}

Keywords: Defects of rail. MFL detection. Signal features. RBF

Abstract. To ensure the security of high speed railway, the basic principle of MFL detection was analyzed. The absolute peak value, the peak-peak value and the peak spacing are selected as the MFL signal features. The relationships between the defect geometry parameters and the MFL signal features are researched by simulation. The method of quantitative recognition defect based on RBF neural network was studied in this paper, and the experimental result shows that the method is feasible.

\section{Introduction}

As the main component of track, railway rail is directly under the pressure of the rail in the railway infrastructure. Defect damage and material degradation will happen in long-term use. The rail defects of stress and fracture and fatigue can lead to the occurrence of accidents, which can result in casualties and property losses. So it is important to test and maintain the real-time and on-line defect of the rail. The effective detection of the defects of the surface and subsurface can be realized by the technology of magnetic flux leakage(MFL) nondestructive testing(NDT) and which don't need coupling agent. Because of those advantages, this technology is widely used in the defects detection of the field of railway track, pipeline and so on. Defect detection can be realized by MFL method, but it is an urgent problem how to realize the quantitative analysis of the defects. In this paper, firstly, the experimental data can be acquired by the existing MFL testing device. Secondly, the defects can be identified by the radial basis function neural network(RBF). At last, the method can be verified by the laboratory data.

\section{The principle of magnetic flux leakage(MFL) detection}

The concept of magnetic flux leakage testing is that the magnetic permeability decreases and the reluctance increases rusulted by the defects of the surface or near the surface, when a magnetic material is magnetized. And the direction of magnetic flux flow also changes. A part of magnetic flux leakes into the air and enters the material again bypass the defects. Magnetic induction sensors response the magnetic flux leaked into the air, which can obtain the information of magnetic flux leakage of the specimen defect. Finally, the detection of defects in the surface and near surface of the rail is realized. Magnetic flux leakage testing belongs to a kind of nondestructive testing technique. The shape and size of the magnetic flux leakage signal varies with the shape and size of the defect.

One road of hall sensors picks up the horizontal component of the defect leakage magnetic field denoted by $\mathrm{X}$, which parallels with an external magnetic field. Another road requires the normal compnent denoted by $\mathrm{Z}$, which perpendiculars to the rail surface. The last road collects the tangential component denoted by $\mathrm{Y}$, which perpendiculars to $\mathrm{X}$ and $\mathrm{Z}$. And then the three-axis MFL detection system can be constituted. Through the analysis of $\mathrm{X}, \mathrm{Y}$ and $\mathrm{Z}$, the relevant information of defects (such as width, depth and angle between the horizontal plane etc.) can be acquired. And then the assessment of the rail health can be realized. 


\section{The feature values of defect}

The MFL detection signal of the rail is formed by the superposition of various interference noise and magnetic leakage signals. The leakage magnetic field will suddenly increase in the crack defect, which will be represented by the local abnormal signal in the waveform of the detection signal. For separating the abnormal signal, and analyzing the defect of the railway, the characteristics of the MFL signal must be analyzed. It is a key step that is the signal feature extraction. Which can be directly impact on the accuracy of the quantitative analysis of defects. In the analysis of the MFL detection signal waveform, their signal features are defned as follows.

The absolute peak value is denoted by Vp. Generally, the peak value of the MFL signal detected around the defect is larger than that of the signal detected in the normal region. But the waveform becomes instability because of noise, surface roughness and other reasons. The absolute peak of the signal can be affected by the baseline fluctuation of the signal. And there may be a false judgment. Vp of $\mathrm{X}$ is shown in the Fig.1.

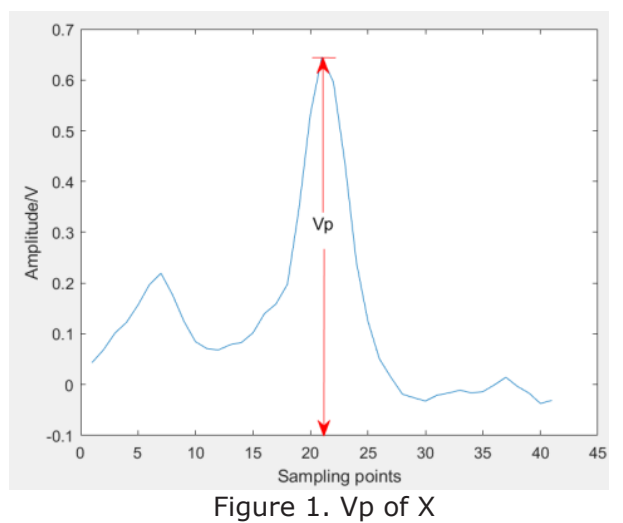

The peak-peak value is denoted by Vp-p. Around the defect signal, the local abnormal signal will appear in the MFL detected signal. And which will has positive and negative peak values. Vp-p is the difference between the positive peak and the negative of that. There is a good corresponding relationship between the peak-peak value of the vertical component and the shape of the defect. Vp-p of $\mathrm{Z}$ is shown in Fig. 2. The peak-peak value overcomes the drawback of the absolute peak value affected by the baseline fluctuation of the signal, So the reliability and accuracy of the identification of the defect signals is increased.

The peak spacing denoted by Dp-p is the distance between the positive peak and the negative of the MFL signal waveform in the analysis of the vertical component. The feature value can provide more accurate information in the analysis of the characteristics of crack width. Dp-p of $\mathrm{Z}$ is also shown in Fig. 2.

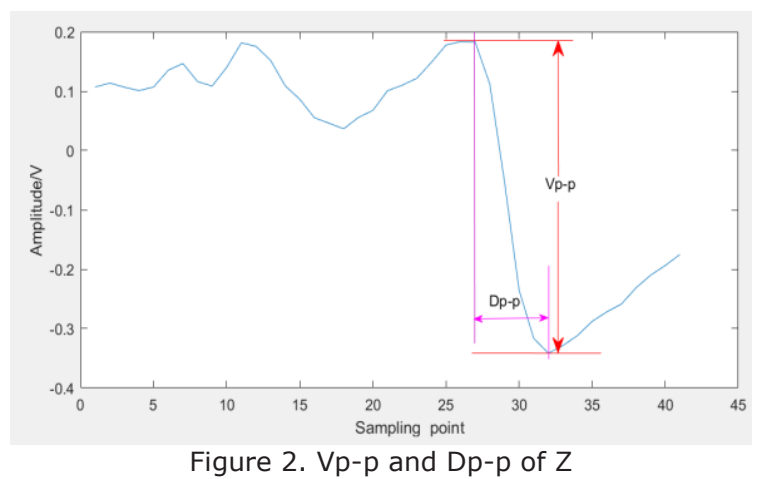

Some information about the defect can be obtained by extracting the characteristic values of the MFL signal and by means of a certain method. For example, Vp and Vp-p are strongly related to the defect depth. Dp-p is strongly related to the defect width. 


\section{Defect recognition system based on the radial basis function (RBF) neural network}

The identification of defect geometrical parameters is difficult point of MFL testing method. Through experiment and consulting data, We got the typical features of MFL signal related to defect geometrical parameters, such as Vp, Vp-p and Dp-p expressed above.The relationship between defect geometrical parameters and MFL signal is nearly definite, but it is difficult to express in function. By using nonlinear approaching ability of the neural network, the nonlinear relation between defect geometrical parameters and MFL signal can be obtained.

The Radial Basis Function Network (RBF) is a kind of feed forward network, which has the characteristics of best approximation and against local minimum problem, and has shorter training period and high forecast accuracy.

\section{Prototype experiment verification and analysis of MFL detected signal}

There are 19 defects in the laboratory rail. 55 defect samples were collected. 40 of them were selected to train the RBF neural network. And another 15 samples verified the network built. True value, training value and error of the training samples are shown in Tab. 1. And rue value, testing value and error of the training samples are shown in Tab. 2. From Table 1, we can see that the error between the trainning results and the actual value is all less than $0.5 \%$. From Table 2, we can see that the error between the testing results and the actual value is less than 5\% except 3 errors. The results can reflect the true value. If the number of the training samples is enough, the flaw shape of the MFL detection signal can be better retrieval by the RBF neural network.

\begin{tabular}{|c|c|c|c|c|c|c|c|c|c|c|c|}
\hline Number of defects & 1 & 2 & 3 & 4 & 5 & 6 & 7 & \multicolumn{2}{|l|}{8} & 9 & 10 \\
\hline Real value(mm) & 4 & 4 & 4 & 4 & 6 & 8 & 4 & \multicolumn{2}{|c|}{4} & 4 & 4 \\
\hline Training value $(\mathrm{mm})$ & 3.994 & 3.9908 & 3.9888 & 3.9876 & 5.9904 & 7.9994 & 3.9912 & \multicolumn{2}{|c|}{3.9908} & 3.9912 & 3.9844 \\
\hline Error $(\%)$ & 0.15 & 0.23 & 0.28 & 0.31 & 0.16 & 0.007 & 0.22 & \multicolumn{2}{|c|}{0.23} & 0.22 & 0.39 \\
\hline Number of defects & 11 & 12 & 13 & 14 & 15 & 16 & 17 & \multicolumn{2}{|l|}{18} & 19 & 20 \\
\hline Real value(mm) & 4 & 4 & 4 & 4 & 4 & 4 & 4 & \multicolumn{2}{|l|}{2} & 4 & 4 \\
\hline Training value (mm) & 3.9997 & 3.9896 & 3.98 & 3.9888 & 3.9848 & 3.9908 & 3.9928 & \multicolumn{2}{|c|}{1.9918} & 3.991 & 3.991 \\
\hline Error $(\%)$ & 0.008 & 0.26 & 0.50 & 0.28 & 0.38 & 0.23 & 0.18 & \multicolumn{2}{|l|}{0.41} & 0.005 & 0.005 \\
\hline Number of defects & 21 & 22 & 23 & 24 & 25 & 26 & 27 & \multicolumn{2}{|l|}{28} & 29 & 30 \\
\hline Real value(mm) & 4 & 4 & 4 & 4 & 4 & 4 & 4 & 4 & & 2 & 6 \\
\hline Training value $(\mathrm{mm})$ & 3.991 & 3.9804 & 3.9998 & 3.9928 & 3.9664 & 3.99 & 3.9916 & 3.9996 & & 1.9999 & 5.9995 \\
\hline Error $(\%)$ & 0.005 & 0.49 & 0.006 & 0.18 & 0.84 & 0.25 & 0.21 & 0.01 & & 0.006 & 0.008 \\
\hline Number of defects & 31 & 32 & 33 & 34 & 35 & 36 & 37 & 38 & & 39 & 40 \\
\hline Real value(mm) & 8 & 4 & 4 & 4 & 4 & 4 & 4 & 4 & & 4 & 4 \\
\hline Training value $(\mathrm{mm})$ & 7.9994 & 3.9910 & 3.994 & 3.9997 & 3.996 & 3.9864 & 3.9997 & 3.9656 & & 3.9908 & 3.9904 \\
\hline Error $(\%)$ & 0.008 & 0.004 & 0.15 & 0.007 & 0.1 & 0.34 & 0.007 & 0.86 & & 0.009 & 0.24 \\
\hline & & & Table 2. & ue value, & ining value & error of test & samples & & & & \\
\hline Number of defects & 1 & 2 & \begin{tabular}{l|l} 
& 3 \\
\end{tabular} & & 4 & 5 & \begin{tabular}{l|l} 
& 6 \\
\end{tabular} & & 7 & & 8 \\
\hline Real value(mm) & 2 & 4 & 4 & & 4 & 4 & 4 & & 4 & & 6 \\
\hline Testing value(mm) & 1.9284 & -4.7304 & & 844 & 3.9704 & 3.9832 & 3.9816 & & 2.642 & & 4.4586 \\
\hline Error $(\%)$ & 3.58 & 218.26 & & & 0.74 & 0.42 & 0.46 & & 33.95 & & 25.69 \\
\hline Number of defects & 9 & 10 & 1 & & 12 & 13 & 14 & & 15 & & \\
\hline Real value(mm) & 4 & 4 & 4 & & 4 & 4 & 4 & & 4 & & \\
\hline Testing value $(\mathrm{mm})$ & 3.9408 & 3.974 & & 968 & 3.8796 & 3.9892 & 3.9999 & & 2.5132 & & \\
\hline Error $(\%)$ & 1.48 & 0.65 & & & 3.01 & 0.27 & 0.002 & & 37.17 & & \\
\hline
\end{tabular}

\section{Conclusions}

Through analysis of magnetic leakage field of MFL inspection, the MFL signal features are defined, they are the peak-peak amplitude and peak spacing value and peak amplitude of the MFL signal. The relationships between the defect geometry parameters and the MFL signal features are researched by simulation.

Considering that it is difficult to identify well casing defect quantitatively, the method of quantitative recognition defect based on RBF neural network was studied in this paper, and which increased the detection accuracy. 


\section{Acknowledgements}

This work was supported in part by the National Science Foundation of China ( 61471191).

\section{References}

[1] Zhang Guoguang, in: Defect Quantitative Recognition Technology of Circumferential Magnetic FluxLeakage Inspection in Pipeline, edited by volume 10 of 2010 International Conerence on Computer Application and System Modeling (ICCASM 2010), p.311-314.

[2] Y. Zhang, Z. F. Ye, C. Wang:A fast method for rectangular crack sizes reconstruction in magnetic flux leakage testing. NDT and EInternational, vol. 42(2009), p. 369-375.

[3] A. Sophian, G. Y. Tian, S. Zairi:Pulsed magnetic flux leakage techniques for crack detection and characterisation.Sensors and Actuators A (Physical). vol. 125(2006), p. 1886-191.

[4] Zhongli Ma, Hongda Liu: Pipeline Defect Dectection and Sizing Based on MFL Data Using Immune FBF Neural Networks.edit by 2007 IEEE Congress on Evolutionary Computation (CEC 2007),p.3399-3403.

[5] Al-Naemi F I, Hall J P, Moses A J: FEM modeling echniques of magnetic flux leakage-type NDT for ferro-magnetic plate inspections[J]. Journal of Magnetism and agnetic Materials, 304(2006),p.790-793.

[6] PAN Hai-fei, WANG Wu-hua: Magnetic flux leakage detection and refutation on defects of high speed rail. CHINA MEASUREMENT \& TEST,vol.39(2013),p.21-24. 